Volume 30 (2021) 203-216

DOI: $10.24330 /$ ieja.969902

\title{
DOMINATION NUMBER IN THE ANNIHILATING-SUBMODULE GRAPH OF MODULES OVER COMMUTATIVE RINGS
}

\author{
Habibollah Ansari-Toroghy and Shokoufeh Habibi \\ Received: 13 June 2020; Revised: 26 November 2020; Accepted: 5 December 2020 \\ Communicated by Sait Halıcıŏ̆lu
}

\begin{abstract}
Let $M$ be a module over a commutative ring $R$. The annihilatingsubmodule graph of $M$, denoted by $A G(M)$, is a simple undirected graph in which a non-zero submodule $N$ of $M$ is a vertex if and only if there exists a nonzero proper submodule $K$ of $M$ such that $N K=(0)$, where $N K$, the product of $N$ and $K$, is denoted by $(N: M)(K: M) M$ and two distinct vertices $N$ and $K$ are adjacent if and only if $N K=(0)$. This graph is a submodule version of the annihilating-ideal graph and under some conditions, is isomorphic with an induced subgraph of the Zariski topology-graph $G\left(\tau_{T}\right)$ which was introduced in [H. Ansari-Toroghy and S. Habibi, Comm. Algebra, 42(2014), 3283-3296]. In this paper, we study the domination number of $A G(M)$ and some connections between the graph-theoretic properties of $A G(M)$ and algebraic properties of module $M$.
\end{abstract}

Mathematics Subject Classification (2020): 13C13, 13C99, 05C75

Keywords: Commutative ring, annihilating-submodule graph, domination number

\section{Introduction}

Throughout this paper $R$ is a commutative ring with a non-zero identity and $M$ is a unital $R$-module. By $N \leq M$ (resp. $N<M$ ) we mean that $N$ is a submodule (resp. proper submodule) of $M$.

Define $\left(N:_{R} M\right)$ or simply $(N: M)=\{r \in R \mid r M \subseteq N\}$ for any $N \leq M$. We denote $((0): M)$ by $A n n_{R}(M)$ or simply $\operatorname{Ann}(M)$. $M$ is said to be faithful if $\operatorname{Ann}(M)=(0)$. Let $N, K \leq M$. Then the product of $N$ and $K$, denoted by $N K$, is defined by $(N: M)(K: M) M$ (see [6]). Define $\operatorname{ann}(N)$ or simply ann $N=\{m \in M \mid$ $m(N: M)=0\}$.

The prime spectrum of $M$ is the set of all prime submodules of $M$ and denoted by $\operatorname{Spec}(M), \operatorname{Max}(M)$ is the set of all maximal submodules of $M$, and $J(M)$, the jacobson radical of $M$, is the intersection of all elements of $\operatorname{Max}(M)$, respectively.

This research was in part supported by a grant from IPM (No. 96130028). 
There are many papers on assigning graphs to rings or modules (see, for example, $[5,7,13,14])$. The annihilating-ideal graph $A G(R)$ was introduced and studied in [14]. $A G(R)$ is a graph whose vertices are ideals of $R$ with nonzero annihilators and in which two vertices $I$ and $J$ are adjacent if and only if $I J=(0)$. Later, it was modified and further studied by many authors (see $[1,2,3,20,22]$ ).

In [7], the present authors introduced and studied the graph $G\left(\tau_{T}\right)$ (resp. $A G(M)$ ), called the Zariski topology-graph (resp. the annihilating-submodule graph), where $T$ is a non-empty subset of $\operatorname{Spec}(M)$.

$A G(M)$ is an undirected simple graph with vertices $V(A G(M))=\{N \leq M \mid$ there exists $(0) \neq K<M$ with $N K=(0)\}$. In this graph, distinct vertices $N, L \in V(A G(M))$ are adjacent if and only if $N L=(0)$ (see [8,9]). Let $A G(M)^{*}$ be the subgraph of $A G(M)$ with vertices $V\left(A G(M)^{*}\right)=\{N<M$ with $(N: M) \neq$ $\operatorname{Ann}(M) \mid$ there exists a submodule $K<M$ with $(K: M) \neq A n n(M)$ and $N K=$ (0)\}. By [7, Theorem 3.4], one conclude that $A G(M)^{*}$ is a connected subgraph. Note that $M$ is a vertex of $A G(M)$ if and only if there exists a nonzero proper submodule $N$ of $M$ with $(N: M)=A n n(M)$ if and only if every nonzero submodule of $M$ is a vertex of $A G(M)$. Clearly, if $M$ is not a vertex of $A G(M)$, then $A G(M)=$ $A G(M)^{*}$. In [10, Lemma 2.8], we showed that under some conditions, $A G(M)$ is isomorphic with an induced subgraph of the Zariski topology-graph $G\left(\tau_{T}\right)$.

In this paper, we study the domination number of $A G(M)$ and some connections between the graph-theoretic properties of $A G(M)$ and algebraic properties of module $M$.

A prime submodule of $M$ is a submodule $P \neq M$ such that whenever $r e \in P$ for some $r \in R$ and $e \in M$, we have $r \in(P: M)$ or $e \in P$ [18].

The notations $Z(R)$ and $N i l(R)$ will denote the set of all zero-divisors, the set of all nilpotent elements of $R$, respectively. Also, $Z_{R}(M)$ or simply $Z(M)$, the set of zero divisors on $M$, is the set $\{r \in R \mid r m=0$ for some $0 \neq m \in M\}$. If $Z(M)=0$, then we say that $M$ is a domain. An ideal $I \leq R$ is said to be nil if $I$ consist of nilpotent elements.

Let us introduce some graphical notions and denotations that are used in what follows: A graph $G$ is an ordered triple $\left(V(G), E(G), \psi_{G}\right)$ consisting of a nonempty set of vertices, $V(G)$, a set $E(G)$ of edges, and an incident function $\psi_{G}$ that associates an unordered pair of distinct vertices with each edge. The edge $e$ joins $x$ and $y$ if $\psi_{G}(e)=\{x, y\}$, and we say $x$ and $y$ are adjacent. The number of edges incident at $x$ in $G$ is called the degree of the vertex $x$ in $G$ and is denoted by $d_{G}(x)$ or simply $d(x)$. A path in graph $G$ is a finite sequence of vertices $\left\{x_{0}, x_{1}, \ldots, x_{n}\right\}$, where $x_{i-1}$ and $x_{i}$ are adjacent for each $1 \leq i \leq n$ and we denote $x_{i-1}-x_{i}$ for existing an edge 
between $x_{i-1}$ and $x_{i}$. The distance between two vertices $x$ and $y$, denoted $d(x, y)$, is the length of the shortest path from $x$ to $y$. The diameter of a connected graph $G$ is the maximum distance between two distinct vertices of $G$. For any vertex $x$ of a connected graph $G$, the eccentricity of $x$, denoted $e(x)$, is the maximum of the distances from $x$ to the other vertices of $G$. The set of vertices with minimum eccentricity is called the center of the graph $G$, and this minimum eccentricity value is the radius of $G$. For some $U \subseteq V(G)$, we denote by $N(U)$, the set of all vertices of $G \backslash U$ adjacent to at least one vertex of $U$ and $N[U]=N(U) \cup\{U\}$.

A graph $H$ is a subgraph of $G$, if $V(H) \subseteq V(G), E(H) \subseteq E(G)$, and $\psi_{H}$ is the restriction of $\psi_{G}$ to $E(H)$. A subgraph $H$ of $G$ is a spanning subgraph of $G$ if $V(H)=V(G)$. A spanning subgraph $H$ of $G$ is called a perfect matching of $G$ if every vertex of $G$ has degree 1 . A subset $S$ of the vertex set $V(G)$ is called independent if no two vertices of $\mathrm{S}$ are adjacent in $\mathrm{G}$.

A clique of a graph is a complete subgraph and the supremum of the sizes of cliques in $G$, denoted by $\operatorname{cl}(G)$, is called the clique number of $G$. Let $\chi(G)$ denote the chromatic number of the graph $G$, that is, the minimal number of colors needed to color the vertices of $G$ so that no two adjacent vertices have the same color. Obviously $\chi(G) \geq \operatorname{cl}(G)$.

A graph $G$ is a split graph if $V(G)$ can be partitioned into two subsets $A$ and $B$ such that the subgraph induced by $A$ in $G$ is a clique in $\mathrm{G}$, and $B$ is an independent subset of $V(G)$.

A subset $D$ of $V(G)$ is called a dominating set if every vertex of $G$ is either in $D$ or adjacent to at least one vertex in $D$. The domination number of $G$, denoted by $\gamma(G)$, is the number of vertices in a smallest dominating set of $G$. A total dominating set of a graph $G$ is a dominating set $S$ such that every vertex is adjacent to a vertex in $S$. The total domination number of $G$, denoted by $\gamma_{t}(G)$, is the minimum cardinality of a total dominating set. A dominating set of cardinality $\gamma(G)\left(\gamma_{t}(G)\right)$ is called a $\gamma$-set $\left(\gamma_{t}\right.$-set). A dominating set $D$ is a connected dominating set if the subgraph $\langle D\rangle$ induced by $D$ is a connected subgraph of $G$. The connected domination number of $G$, denoted by $\gamma_{c}(G)$, is the minimum cardinality of a connected dominating set of $G$. A dominating set $D$ is a clique dominating set if the subgraph $\langle D\rangle$ induced by $D$ is complete in $G$. The clique domination number $\gamma_{c l}(G)$ of $G$ equals the minimum cardinality of a clique dominating set of $G$. A dominating set $D$ is a paired-dominating set if the subgraph $\langle D\rangle$ induced by $D$ has a perfect matching. The paired-domination number $\gamma_{p r}(G)$ of $G$ equals the minimum cardinality of a paired-dominating set of $G$. 
A vertex $u$ is a neighbor of $v$ in $G$, if $u v$ is an edge of $G$, and $u \neq v$. The set of all neighbors of $v$ is the open neighborhood of $v$ or the neighbor set of $v$, and is denoted by $N(v)$; the set $N[v]=N(v) \cup\{v\}$ is the closed neighborhood of $v$ in $G$.

Let $S$ be a dominating set of a graph $G$, and $u \in S$. The private neighborhood of $u$ relative to $S$ in $G$ is the set of vertices which are in the closed neighborhood of $u$, but not in the closed neighborhood of any vertex in $S \backslash\{u\}$. Thus the private neighborhood $P_{N}(u, S)$ of $u$ with respect to $S$ is given by $P_{N}(u, S)=$ $N[u] \backslash\left(\cup_{v \in S \backslash\{u\}} N[v]\right)$. A set $S \subseteq V(G)$ is called irredundant if every vertex $v$ of $S$ has at least one private neighbor. An irredundant set $S$ is a maximal irredundant set if for every vertex $u \in V \backslash S$, the set $S \cup\{u\}$ is not irredundant. The irredundance number $\operatorname{ir}(G)$ is the minimum cardinality of maximal irredundant sets. There are so many domination parameters in the literature and for more details one can refer [16].

A bipartite graph is a graph whose vertices can be divided into two disjoint sets $U$ and $V$ such that every edge connects a vertex in $U$ to one in $V$; that is, $U$ and $V$ are each independent sets and is denoted by $B_{n, m}$, where $V$ and $U$ are of size $n$ and $m$, respectively. A complete bipartite graph on $n$ and $m$ vertices, denoted by $K_{n, m}$, where $V$ and $U$ are of size $n$ and $m$, respectively, and $E(G)$ connects every vertex in $V$ with all vertices in $U$. Note that a graph $K_{1, m}$ is called a star graph and the vertex in the singleton partition is called the center of the graph. We denote by $C_{n}$ and $P_{n}$ a cycle and a path of order $n$, respectively (see [15]).

In Section 2, a dominating set of $A G(M)$ is constructed using elements of the center when $M$ is an Artinian module. Also we prove that the domination number of $A G(M)$ is equal to the number of factors in the Artinian decomposition of $M$ and we also find several domination parameters of $A G(M)$. In Section 3, some relations between the domination numbers and the total domination numbers of annihilating-submodule graphs are studied. Also, we study the domination number of the annihilating-submodule graphs for reduced rings with finitely many minimal primes and faithful modules.

The following results are useful for further reference in this paper.

Proposition 1.1. Suppose that $e$ is an idempotent element of $R$. We have the following statements.

(a) $R=R_{1} \times R_{2}$, where $R_{1}=e R$ and $R_{2}=(1-e) R$.

(b) $M=M_{1} \times M_{2}$, where $M_{1}=e M$ and $M_{2}=(1-e) M$.

(c) For every submodule $N$ of $M, N=N_{1} \times N_{2}$ such that $N_{1}$ is an $R_{1}$ submodule $M_{1}, N_{2}$ is an $R_{2}$-submodule $M_{2}$, and $\left(N:_{R} M\right)=\left(N_{1}:_{R_{1}}\right.$ $\left.M_{1}\right) \times\left(N_{2}:_{R_{2}} M_{2}\right)$. 
(d) For submodules $N$ and $K$ of $M, N K=N_{1} K_{1} \times N_{2} K_{2}$ such that $N=$ $N_{1} \times N_{2}$ and $K=K_{1} \times K_{2}$.

(e) Prime submodules of $M$ are $P \times M_{2}$ and $M_{1} \times Q$, where $P$ and $Q$ are prime submodules of $M_{1}$ and $M_{2}$, respectively.

Proof. This is clear.

We need the following results.

Lemma 1.2. (See $\left[4\right.$, Proposition 7.6].) Let $R_{1}, R_{2}, \ldots, R_{n}$ be non-zero ideals of $R$. Then the following statements are equivalent:

(a) $R=R_{1} \times \ldots \times R_{n}$;

(b) As an abelian group $R$ is the direct sum of $R_{1}, \ldots, R_{n}$;

(c) There exist pairwise orthogonal idempotents $e_{1}, \ldots, e_{n}$ with $1=e_{1}+\ldots+e_{n}$, and $R_{i}=R e_{i}, i=1, \ldots, n$.

Lemma 1.3. (See [17, Theorem 21.28].) Let $I$ be a nil ideal in $R$ and $u \in R$ be such that $u+I$ is an idempotent in $R / I$. Then there exists an idempotent e in $u R$ such that $e-u \in I$.

Lemma 1.4. (See [9, Lemma 2.4].) Let $N$ be a minimal submodule of $M$ and let Ann $(M)$ be a nil ideal. Then we have $N^{2}=(0)$ or $N=e M$ for some idempotent $e \in R$.

Proposition 1.5. Let $R / A n n(M)$ be an Artinian ring and let $M$ be a finitely generated module. Then every nonzero proper submodule $N$ of $M$ is a vertex in $A G(M)$.

Theorem 1.6. (See $[9$, Theorem 2.5].) Let Ann $(M)$ be a nil ideal. There exists a vertex of $A G(M)$ which is adjacent to every other vertex if and only if $M=$ $e M \oplus(1-e) M$, where $e M$ is a simple module and $(1-e) M$ is a prime module for some idempotent $e \in R$, or $Z(M)=\operatorname{Ann}((N: M) M)$, where $N$ is a nonzero proper submodule of $M$ or $M$ is a vertex of $A G(M)$.

Theorem 1.7. (See [9, Theorem 3.3].) Let $M$ be a faithful module. Then the following statements are equivalent.

(a) $\chi\left(A G(M)^{*}\right)=2$.

(b) $A G(M)^{*}$ is a bipartite graph with two nonempty parts.

(c) $A G(M)^{*}$ is a complete bipartite graph with two nonempty parts.

(d) Either $R$ is a reduced ring with exactly two minimal prime ideals, or $A G(M)^{*}$ is a star graph with more than one vertex. 
Corollary 1.8. (See [9, Corollary 3.5].) Let $R$ be a reduced ring and assume that $M$ is a faithful module. Then the following statements are equivalent.

(a) $\chi\left(A G(M)^{*}\right)=2$.

(b) $A G(M)^{*}$ is a bipartite graph with two nonempty parts.

(c) $A G(M)^{*}$ is a complete bipartite graph with two nonempty parts.

(d) $R$ has exactly two minimal prime ideals.

Theorem 1.9. (See [8, Theorem 2.7].) If $A G(M)$ is a tree, then either $A G(M)$ is a star graph or $A G(M) \cong P_{4}$. Moreover, $A G(M) \cong P_{4}$ if and only if $M=F \times S$, where $F$ is a simple module and $S$ is a module with a unique non-trivial submodule.

Proposition 1.10. (See [16, Proposition 3.9].) Every minimal dominating set in a graph $G$ is a maximal irredundant set of $G$.

\section{Domination number in the annihilating-submodule graph for Artinian modules}

The main goal in this section, is to obtain the value certain domination parameters of the annihilating-submodule graph for Artinian modules.

Recall that $M$ is a vertex of $A G(M)$ if and only if there exists a nonzero proper submodule $N$ of $M$ with $(N: M)=\operatorname{Ann}(M)$ if and only if every nonzero submodule of $M$ is a vertex of $A G(M)$. In this case, the vertex $N$ is adjacent to every other vertex. Hence $\gamma(A G(M))=1$ and $\gamma_{t}(A G(M))=2$. So we assume that throughout this paper $M$ is not a vertex of $A G(M)$. Clearly, if $M$ is not a vertex of $A G(M)$, then $A G(M)=A G(M)^{*}$.

We start with the following remark which completely characterizes all modules for which $\gamma(A G(M))=1$.

Remark 2.1. Let $\operatorname{Ann}(M)$ be a nil ideal. By Theorem 1.6, there exists a vertex of $A G(M)$ which is adjacent to every other vertex if and only if $M=e M \oplus(1-e) M$, where $e M$ is a simple module and $(1-e) M$ is a prime module for some idempotent $e \in R$, or $Z(M)=\operatorname{Ann}((N: M) M)$, where $N$ is a nonzero proper submodule of $M$ or $M$ is a vertex of $A G(M)$. Now, let $A n n(M)$ be a nil ideal and $M$ be a domain module. Then $\gamma(A G(M))=1$ if and only if $M=e M \oplus(1-e) M$, where $e M$ is a simple module and $(1-e) M$ is a prime module for some idempotent $e \in R$.

Theorem 2.2. Let $M$ be a finitely generated Artinian local module. Assume that $N$ is the unique maximal submodule of $M$. Then the radius of $A G(M)$ is 0 or 1 and the center of $A G(M)$ is $\{K \subseteq \operatorname{ann}(N) \mid K \neq(0)$ is a submodule in $M\}$. 
Proof. If $N$ is the only non-zero proper submodule of $M$, then $A G(M) \cong K_{1}$, $e(N)=0$ and the radius of $A G(M)$ is 0 . Assume that the number of non-zero proper submodules of $M$ is greater than 1 . Since $M$ is finitely generated Artinian module, there exists $m \in \mathbb{N}, m>1$ such that $N^{m}=(0)$ and $N^{m-1} \neq(0)$. For any non-zero submodule $K$ of $M, K N^{m-1} \subseteq N N^{m-1}=(0)$ and so $d\left(N^{m-1}, K\right)=1$. Hence $e\left(N^{m-1}\right)=1$ and so the radius of $A G(M)$ is 1 . Suppose $K$ and $L$ are arbitrary non-zero submodules of $M$ and $K \subseteq \operatorname{ann}(N)$. Then $K L \subseteq K N=(0)$ and hence $e(K)=1$. Suppose $(0) \neq K^{\prime} \nsubseteq \operatorname{ann}(N)$. Then $K^{\prime} N \neq(0)$ and so $e\left(K^{\prime}\right)>1$. Hence the center of $A G(M)$ is $\{K \subseteq \operatorname{ann}(N) \mid K \neq(0)$ is a submodule in $M\}$.

Corollary 2.3. Let $M$ be a finitely generated Artinian local module and $N$ is the unique maximal submodule of $M$. Then the following hold good.

(a) $\gamma(A G(M))=1$.

(b) $D$ is a $\gamma$-set of $A G(M)$ if and only if $D \subseteq \operatorname{ann}(N)$.

Proof. (a) Trivial from Theorem 2.2.

(b) Let $D=\{K\}$ be a $\gamma$-set of $A G(M)$. Suppose $K \nsubseteq \operatorname{ann}(N)$. Then $K N \neq(0)$ and so $N$ is not dominated by $K$, a contradiction. Conversely, suppose $D \subseteq \operatorname{ann}(N)$. Let $K$ be an arbitrary vertex in $A G(M)$. Then $K L \subseteq N L=(0)$ for every $L \in D$. i.e., every vertex $K$ is adjacent to every $L \in D$. If $|D|>1$, then $D \backslash\left\{L^{\prime}\right\}$ is also a dominating set of $A G(M)$ for some $L^{\prime} \in D$ and so $D$ is not minimal. Thus $|D|=1$ and so $D$ is a $\gamma$-set by $(a)$.

Theorem 2.4. Let $M=\oplus_{i=1}^{n} M_{i}$, where $M_{i}$ is a finitely generated Artinian local module for all $1 \leq i \leq n$ and $n \geq 2$. Then the radius of $A G(M)$ is 2 and the center of $A G(M)$ is $\{K \subseteq J(M) \mid K \neq(0)$ is a submodule in $M\}$.

Proof. Let $M=\oplus_{i=1}^{n} M_{i}$, where $M_{i}$ is a finitely generated Artinian local module for all $1 \leq i \leq n$ and $n \geq 2$. Let $J_{i}$ be the unique maximal submodule in $M_{i}$ with nilpotency $n_{i}$. Note that $\operatorname{Max}(M)=\left\{N_{1}, \ldots, N_{n} \mid N_{i}=M_{1} \oplus \ldots \oplus M_{i-1} \oplus\right.$ $\left.J_{i} \oplus M_{i+1} \oplus \ldots \oplus M_{n}, 1 \leq i \leq n\right\}$ is the set of all maximal submodules in $M$. Consider $D_{i}=(0) \oplus \ldots \oplus(0) \oplus J_{i}^{n_{i}-1} \oplus(0) \oplus \ldots \oplus(0)$ for $1 \leq i \leq n$. Note that $J(M)=J_{1} \oplus \ldots \oplus J_{n}$ is the Jacobson radical of $M$ and any non-zero submodule in $M$ is adjacent to $D_{i}$ for some $i$. Let $K$ be any non-zero submodule of $M$. Then $K=\oplus_{i=1}^{n} K_{i}$, where $K_{i}$ is a submodule of $M_{i}$.

Case 1. If $K=N_{i}$ for some $i$, then $K D_{j} \neq(0)$ and $K N_{j} \neq(0)$ for all $j \neq i$. Note that $N(K)=\left\{(0) \oplus \ldots \oplus(0) \oplus L_{i} \oplus(0) \oplus \ldots \oplus(0) \mid J_{i} L_{i}=(0), L_{i}\right.$ is a nonzero submodule in $\left.M_{i}\right\}$. Clearly $N(K) \cap N\left(N_{j}\right)=(0), d\left(K, N_{j}\right) \neq 2$ for all $j \neq i$, and so $K-D_{i}-D_{j}-N_{j}$ is a path in $A G(M)$. Therefore $e(K)=3$ and so $e(N)=3$ 
for all $N \in \operatorname{Max}(M)$.

Case 2. If $K \neq D_{i}$ and $K_{i} \subseteq J_{i}$ for all $i$. Then $K D_{i}=(0)$ for all $i$. Let $L$ be any non-zero submodule of $M$ with $K L \neq(0)$. Then $L D_{j}=(0)$ for some $j, K-D_{j}-L$ is a path in $A G(M)$ and so $e(K)=2$.

Case 3. If $K_{i}=M_{i}$ for some $i$, then $K D_{i} \neq(0), K N_{i} \neq(0)$ and $K D_{j}=(0)$ for some $j \neq i$. Thus $K-D_{j}-D_{i}-N_{i}$ is a path in $A G(M), d\left(K, N_{i}\right)=3$ and so $e(K)=3$. Thus $e(K)=2$ for all $K \subseteq J(M)$. Further note that in all the cases center of $A G(M)$ is $\{K \subseteq J(M) \mid K \neq(0)$ is a submodule in $M\}$.

In view of Theorems 2.2 and 2.4, we have the following corollary.

Corollary 2.5. Let $M=\oplus_{i=1}^{n} M_{i}$, where $M_{i}$ is a simple module for all $1 \leq i \leq n$ and $n \geq 2$. Then the radius of $A G(M)$ is 1 or 2 and the center of $A G(M)$ is $\cup_{i=1}^{n} D_{i}$, where $D_{i}=(0) \oplus \ldots \oplus(0) \oplus M_{i} \oplus(0) \oplus \ldots \oplus(0)$ for $1 \leq i \leq n$.

Proposition 2.6. Let $M=\oplus_{i=1}^{n} M_{i}$, where $M_{i}$ is a finitely generated Artinian local module for all $1 \leq i \leq n$ and $n \geq 2\left(M \neq M_{1} \oplus M_{2}\right.$, where $M_{1}$ and $M_{2}$ are simple modules). Then

(a) $\gamma(A G(M))=n$.

(b) $\operatorname{ir}(A G(M))=n$.

(c) $\gamma_{c}(A G(M))=n$.

(d) $\gamma_{t}(A G(M))=n$.

(e) $\gamma_{c l}(A G(M))=n$.

(f) $\gamma_{p r}(A G(M))=n$, if $n$ is even and $\gamma_{p r}(A G(M))=n+1$, if $n$ is odd.

Proof. Let $J_{i}$ be the unique maximal submodule in $M_{i}$ with nilpotency $n_{i}$. Let $\Omega=\left\{D_{1}, D_{2}, \ldots, D_{n}\right\}$, where $D_{i}=(0) \oplus \ldots \oplus(0) \oplus J_{i}^{n_{i}-1} \oplus(0) \oplus \ldots \oplus(0)$ for $1 \leq i \leq n$. Note that any non-zero submodule in $M$ is adjacent to $D_{i}$ for some $i$. Therefore $N[\Omega]=V(A G(M)), \Omega$ is a dominating set of $A G(M)$ and so $\gamma(A G(M)) \leq n$. Suppose $S$ is a dominating set of $A G(M)$ with $|S|<n$. Then there exists $N \in \operatorname{Max}(M)$ such that $N K \neq(0)$ for all $K \in S$, a contradiction. Hence $\gamma(A G(M))=n$. By Proposition 1.10, $\Omega$ is a maximal irredundant set with minimum cardinality and so $\operatorname{ir}(A G(M))=n$. Clearly $\langle\Omega\rangle$ is a complete subgraph of $A G(M)$. Hence $\gamma_{c}(A G(M))=\gamma_{t}(A G(M))=\gamma_{c l}(A G(M))=n$. If $n$ is even, then $\langle\Omega>$ has a perfect matching and so $\Omega$ is a paired-dominating set of $A G(M)$. Thus $\gamma_{p r}(A G(M))=n$. If $n$ is odd, then $<\Omega \cup K>$ has a perfect matching for some $K \in V(A G(M)) \backslash \Omega$. and so $\Omega \cup K$ is a paired-dominating set of $A G(M)$. Thus $\gamma_{p r}(A G(M))=n$ if $n$ even and $\gamma_{p r}(A G(M))=n+1$ if $n$ is odd. 
Let $M=\oplus_{i=1}^{n} M_{i}$, where $M_{i}$ is a finitely generated Artinian local module for all $1 \leq i \leq n$ and $n \geq 2$. Then by Theorem 2.4, radius of $A G(M)$ is 2 . Further, by Proposition 2.6, the domination number of $A G(M)$ is equal to $n$, where $n$ is the number of distinct maximal submodules of $M$. However, this need not be true if the radius of $A G(M)$ is 1 . For, consider the ring $M=M_{1} \oplus M_{2}$, where $M_{1}$ and $M_{2}$ are simple modules. Then $A G(M)$ is a star graph and so has radius 1, whereas $M$ has two distinct maximal submodules. The following corollary shows that a more precise relationship between the domination number of $A G(M)$ and the number of maximal submodules in $M$, when $M$ is finite.

Corollary 2.7. Let $M$ be a finitely generated Artinian module, $M$ is a faithful module, and $\gamma(A G(M))=n$. Then either $M=M_{1} \oplus M_{2}$, where $M_{1}$ and $M_{2}$ are simple modules or $M$ has $n$ maximal submodules.

Proof. When $\gamma(A G(M))=1$, the proof follows from [9, Corollary 2.12]. If $\gamma(A G(M))=n$, where $n \geq 2$, then $M$ can not be $M=M_{1} \oplus M_{2}$, where $M_{1}$ and $M_{2}$ are simple modules. Hence $M=\oplus_{i=1}^{m} M_{i}$, where $M_{i}$ is a finitely generated Artinian local module for all $1 \leq i \leq m$ and $m \geq 2$. By Proposition 2.6, $\gamma(A G(M))=m$. Hence by assumption $m=n$, i.e., $M=\oplus_{i=1}^{n} M_{i}$, where $M_{i}$ is a finitely generated Artinian local module for all $1 \leq i \leq n$ and $n \geq 2$. One can see now that $M$ has $n$ maximal submodules.

\section{The relationship between $\gamma_{t}(A G(M))$ and $\gamma(A G(M))$}

The main goal in this section is to study the relation between $\gamma_{t}(A G(M))$ and $\gamma(A G(M))$.

Theorem 3.1. Let $M$ be a module. Then

$$
\gamma_{t}(A G(M))=\gamma(A G(M)) \text { or } \gamma_{t}(A G(M))=\gamma(A G(M))+1 .
$$

Proof. Assume that $\gamma_{t}(A G(M)) \neq \gamma(A G(M))$ and $D$ is a $\gamma$-set of $A G(M)$. If $\gamma(A G(M))=1$, then it is clear that $\gamma_{t}(A G(M))=2$. So let $\gamma(A G(M))>1$ and put $k=\operatorname{Max}\left\{n \mid\right.$ there exist $L_{1}, \ldots, L_{n} \in D$ such that $\left.\sqcap_{i=1}^{n} L_{i} \neq 0\right\}$. Since $\gamma_{t}(A G(M)) \neq \gamma(A G(M))$, we have $k \geq 2$. Let $L_{1}, \ldots, L_{k} \in D$ be such that $\sqcap_{i=1}^{k} L_{i} \neq 0$. Then $S=\left\{\sqcap_{i=1}^{k} L_{i}\right.$, ann $L_{1}, \ldots$, ann $\left.L_{k}\right\} \cup D \backslash\left\{L_{1}, \ldots, L_{k}\right\}$ is a $\gamma_{t}$-set. Hence $\gamma_{t}(A G(M))=\gamma(A G(M))+1$.

Example 3.2. Let $C_{n}$ and $P_{n}$ be a cycle and a path with $n$ vertices, respectively.

(a) Clearly, $\gamma\left(C_{n}\right)=\gamma\left(P_{n}\right)=[n / 3]$ (see [19, Example 1]).

(b) Let $\mathbb{Z}_{2} \times \mathbb{Z}_{3}$ as $\mathbb{Z}_{12}$-module. It is easy to see that $A G\left(\mathbb{Z}_{2} \times \mathbb{Z}_{3}\right)=P_{2}$ and $\gamma_{t}\left(P_{2}\right)=2=\gamma\left(P_{2}\right)+1$. 
(c) By [12, Lemma 10.9.5], for any split graph $G, \gamma_{t}(G)=\gamma(G)$. Let $\mathbb{Z}_{3} \times \mathbb{Z}_{4}$ as $\mathbb{Z}_{24}$-module. The split graph $A G\left(\mathbb{Z}_{3} \times \mathbb{Z}_{4}\right)=P_{4}$ and $\gamma_{t}\left(P_{4}\right)=\gamma\left(P_{4}\right)=2$.

In the following result we find the total domination number of $A G(M)$.

Theorem 3.3. Let $S$ be the set of all maximal elements of the set $V(A G(M))$. If $|S|>1$, then $\gamma_{t}(A G(M))=|S|$.

Proof. Suppose that $S$ is the set of all maximal elements of the set $V(A G(M))$. Let $K \in S$. First we show that $K=\operatorname{ann}(\operatorname{annK})$ and there exists $m \in M$ such that $K=\operatorname{ann}(R m)$. Since $\operatorname{ann} K \neq 0$, there exists $0 \neq m \in \operatorname{annK}$. Hence $K \subseteq \operatorname{ann}(\operatorname{annK}) \subseteq \operatorname{ann}(R m)$. Thus by the maximality of $K$, we have $K=\operatorname{ann}(\operatorname{ann} K)=\operatorname{ann}(R m)$. For any $K \in S$, choose $m_{K} \in M$ such that $K=\operatorname{ann}\left(R m_{K}\right)$. We assert that $D=\left\{R m_{K} \mid K \in S\right\}$ is a total dominating set of $A G(M)$. Since for every $L \in V(A G(M))$ there exists $K \in S$ such that $L \subseteq K=\operatorname{ann}\left(R m_{K}\right), L$ and $R m_{K}$ are adjacent. Also for each pair $K, K^{\prime} \in S$, we have $\left(R m_{K}\right)\left(R m_{K^{\prime}}\right)=0$. Namely, if there exists $m \in\left(R m_{K}\right)\left(R m_{K^{\prime}}\right) \backslash\{0\}$, then $K=K^{\prime}=\operatorname{ann}(R m)$. Thus $\gamma_{t}(A G(M)) \leq|S|$. To complete the proof, we show that each element of an arbitrary $\gamma_{t}$-set of $A G(M)$ is adjacent to exactly one element of $S$. Assume to the contrary, that a vertex $L^{\prime}$ of a $\gamma_{t}$-set of $A G(M)$ is adjacent to $K$ and $K^{\prime}$, for $K, K^{\prime} \in S$. Thus $K=K^{\prime}=a n n L^{\prime}$, which is impossible. Therefore $\gamma_{t}(A G(M))=|S|$.

The following corollary is a connection between Sections 2 and 3.

Corollary 3.4. Let $M=\oplus_{i=1}^{n} M_{i}$, where $M_{i}$ is a finitely generated Artinian local module for all $1 \leq i \leq n, n \geq 2\left(M \neq M_{1} \oplus M_{2}\right.$, where $M_{1}$ and $M_{2}$ are simple modules). Then $\gamma_{t}(A G(M))=\gamma(A G(M))=|\operatorname{Max}(M)|$.

Proof. Let $M=\oplus_{i=1}^{n} M_{i}$, where $\left(M_{i}, J_{i}\right)$ is a finitely generated Artinian local module for all $1 \leq i \leq n$ and $n \geq 2$. Recall that $\operatorname{Max}(M)=\left\{N_{1}, \ldots, N_{n} \mid\right.$ $\left.N_{i}=M_{1} \oplus \ldots \oplus M_{i-1} \oplus J_{i} \oplus M_{i+1} \oplus \ldots \oplus M_{n}, 1 \leq i \leq n\right\}$. By Proposition 1.5 , every nonzero proper submodule of $M$ is a vertex in $A G(M)$. So the set of maximal elements of $V(A G(M))$ and $\operatorname{Max}(M)$ are equal and hence by Theorem $3.3, \gamma_{t}(A G(M))=|\operatorname{Max}(M)|$. Finally, the result follows from Proposition 2.6.

Example 3.5. Let $\mathbb{Z}_{3} \times \mathbb{Z}_{4}$ as $\mathbb{Z}_{24}$-module. $S=\left\{(0) \times \mathbb{Z}_{4}, \mathbb{Z}_{3} \times \overline{2} \mathbb{Z}_{4}\right\}$ is the set of all maximal elements of $A G\left(\mathbb{Z}_{3} \times \mathbb{Z}_{4}\right)$ and $\gamma_{t}\left(A G\left(\mathbb{Z}_{3} \times \mathbb{Z}_{4}\right)\right)=\gamma_{t}\left(P_{4}\right)=2=|S|$.

Theorem 3.6. Let $R$ be a reduced ring, $M$ is a faithful module, and $|\operatorname{Min}(R)|<\infty$. If $\gamma(A G(M))>1$, then $\gamma_{t}(A G(M))=\gamma(A G(M))=|\operatorname{Min}(R)|$. 
Proof. Since $R$ is reduced, $M$ is a faithful module, and $\gamma(A G(M))>1$, we have $|\operatorname{Min}(R)|>1$. Suppose that $\operatorname{Min}(R)=\left\{p_{1}, \ldots, p_{n}\right\}$. If $n=2$, the result follows from Corollary 1.8. Therefore, suppose that $n \geq 3$. We define $\widehat{p_{i} M}=$ $p_{1} \ldots p_{i-1} p_{i+1} \ldots p_{n} M$, for every $i=1, \ldots, n$. Clearly, $\widehat{p_{i} M} \neq 0$, for every $i=$ $1, \ldots, n$. Since $R$ is reduced, we deduce that $\widehat{p_{i} M} p_{i} M=0$. Therefore, every $p_{i} M$ is a vertex of $A G(M)$. If $K$ is a vertex of $A G(M)$, then by [11, Corollary 3.5], $(K: M) \subseteq Z(R)=\cup_{i=1}^{n} p_{i}$. It follows from the Prime Avoidance Theorem that $(K: M) \subseteq p_{i}$, for some $i, 1 \leq i \leq n$. Thus $p_{i} M$ is a maximal element of $V(A G(M))$, for every $i=1, \ldots, n$. From Theorem 3.3, $\gamma_{t}(A G(M))=|\operatorname{Min}(R)|$. Now, we show that $\gamma(A G(M))=n$. Assume to the contrary, that $B=\left\{J_{1}, \ldots, J_{n-1}\right\}$ is a dominating set for $A G(M)$. Since $n \geq 3$, the submodules $p_{i} M$ and $p_{j} M$, for $i \neq j$ are not adjacent (from $p_{i} p_{j}=0 \subseteq p_{k}$ it would follow that $p_{i} \subseteq p_{k}$ or $p_{j} \subseteq p_{k}$ which is not true). Because of that, we may assume that for some $k<n-1, J_{i}=p_{i} M$ for $i=1, \ldots, k$, but none of the other of submodules from $B$ are equal to some $p_{s} M$ (if $B=\left\{p_{1} M, \ldots, p_{n-1} M\right\}$, then $p_{n} M$ would be adjacent to some $p_{i} M$, for $\left.i \neq n\right)$. So every submodule in $\left\{p_{k+1} M, \ldots, p_{n} M\right\}$ is adjacent to a submodule in $\left\{J_{k+1}, \ldots, J_{n-1}\right\}$. It follows that for some $s \neq t$, there is an $l$ such that $\left(p_{s} M\right) J_{l}=0=\left(p_{t} M\right) J_{l}$. Since $p_{s} \nsubseteq p_{t}$, it follows that $J_{l} \subseteq p_{t} M$, so $J_{l}^{2}=0$, which is impossible, since the $\operatorname{ring} R$ is reduced. So $\gamma_{t}(A G(M))=\gamma(A G(M))=|\operatorname{Min}(R)|$.

Theorem 3.3 leads to the following corollary.

Corollary 3.7. Let $R$ be a reduced ring, $M$ is a faithful module, and $|\operatorname{Min}(R)|<$ $\infty$. If $\gamma(A G(M))>1$, then the following are equivalent.
(a) $\gamma(A G(M))=2$.
(b) $A G(M)=B_{n, m}$ such that $n, m \geq 2$.
(c) $A G(M)=K_{n, m}$ such that $n, m \geq 2$.
(d) $R$ has exactly two minimal primes.

Proof. Follows from Theorem 3.3 and Corollary 1.8.

In the following theorem the domination number of bipartite annihilating-submodule graphs is given.

Theorem 3.8. Let $M$ be a faithful module. If $A G(M)$ is a bipartite graph, then $\gamma(A G(M)) \leq 2$.

Proof. Let $M$ be a faithful module. If $A G(M)$ is a bipartite graph, then from Theorem 1.7, either $R$ is a reduced ring with exactly two minimal prime ideals, or $A G(M)$ is a star graph with more than one vertex. If $R$ is a reduced ring with 
exactly two minimal prime ideals and $\gamma(A G(M))=1$, then we are done. If $R$ is a reduced ring with exactly two minimal prime ideals and $\gamma(A G(M))>1$, then the result follows by Corollary 3.7. If $A G(M)$ is a star graph with more than one vertex, then we are done.

Theorem 3.9. If $R$ is a Notherian ring and $M$ a finitely generated module, then $\gamma(A G(M)) \leq|A s s(M)|<\infty$.

Proof. By [21], Since $R$ is a Notherian ring and $M$ a finitely generated module, $|A s s(M)|<\infty$. Let $\operatorname{Ass}(M)=\left\{p_{1}, \ldots, p_{n}\right\}$, where $p_{i}=\operatorname{ann}\left(m_{i}\right)$ for some $m_{i} \in$ $M$ for every $i=1, \ldots, n$. Set $A=\left\{R m_{i} \mid 1 \leq i \leq n\right\}$. We show that $A$ is a dominating set of $A G(M)$. Clearly, every $R m_{i}$ is a vertex of $A G(M)$, for $i=1, \ldots, n$ $\left(\left(p_{i} M\right)\left(m_{i} R\right)=0\right)$. If $K$ is a vertex of $A G(M)$, then [21, Corollary 9.36] implies that $(K: M) \subseteq Z(M)=\cup_{i=1}^{n} p_{i}$. It follows from the Prime Avoidance Theorem that $(K: M) \subseteq p_{i}$, for some $i, 1 \leq i \leq n$. Thus $K\left(R m_{i}\right)=0$, as desired.

The remaining result of this paper provides the domination number of the annihilating-submodule graph of a finite direct product of modules.

Theorem 3.10. For a module $M$, which is a product of two (nonzero) modules, one of the following holds.

(a) If $M \cong F \times D$, where $F$ is a simple module and $D$ is a prime module, then $\gamma(A G(M))=1$.

(b) If $M \cong D_{1} \times D_{2}$, where $D_{1}$ and $D_{2}$ are prime modules which are not simple, then $\gamma(A G(M))=2$.

(c) If $M \cong M_{1} \times D$, where $M_{1}$ is a module which is not prime and $D$ is a prime module, then $\gamma(A G(M))=\gamma\left(A G\left(M_{1}\right)\right)+1$.

(d) If $M \cong M_{1} \times M_{2}$, where $M_{1}$ and $M_{2}$ are two modules which are not prime, then $\gamma(A G(M))=\gamma\left(A G\left(M_{1}\right)\right)+\gamma\left(A G\left(M_{2}\right)\right)$.

Proof. Parts (a) and (b) are trivial.

(c) Without loss of generality, one can assume that $\gamma\left(A G\left(M_{1}\right)\right)<\infty$. Suppose that $\gamma\left(A G\left(M_{1}\right)\right)=n$ and $\left\{K_{1}, \ldots, K_{n}\right\}$ is a minimal dominating set of $A G\left(M_{1}\right)$. It is not hard to see that $\left\{K_{1} \times 0, \ldots, K_{n} \times 0,0 \times D\right\}$ is the smallest dominating set of $A G(M)$.

(d) We may assume that $\gamma\left(A G\left(M_{1}\right)\right)=m$ and $\gamma\left(A G\left(M_{2}\right)\right)=n$, for some positive integers $m$ and $n$. Let $\left\{K_{1}, \ldots, K_{m}\right\}$ and $\left\{L_{1}, \ldots, L_{n}\right\}$ be two minimal dominating sets in $A G\left(M_{1}\right)$ and $A G\left(M_{2}\right)$, respectively. It is easy to see that $\left\{K_{1} \times 0, \ldots, K_{m} \times\right.$ $\left.0,0 \times L_{1} \ldots 0 \times L_{n}\right\}$ is the smallest dominating set in $A G(M)$. 
Acknowledgement. The authors would like to thank the referee for his/her very helpful comments and valuable suggestions.

\section{References}

[1] G. Aalipour, S. Akbari, R. Nikandish, M. J. Nikmehr and F. Shaveisi, On the coloring of the annihilating-ideal graph of a commutative ring, Discrete Math., 312(17) (2012), 2620-2626.

[2] G. Aalipour, S. Akbari, R. Nikandish, M. J. Nikmehr and F. Shaveisi, Minimal prime ideals and cycles in annihilating-ideal graphs, Rocky Mountain J. Math., 43(5) (2013), 1415-1425.

[3] G. Aalipour, S. Akbari, M. Behboodi, R. Nikandish, M. J. Nikmehr and F. Shaveisi, The classification of the annihilating-ideal graphs of commutative rings, Algebra Colloq., 21(2) (2014), 249-256.

[4] F. W. Anderson and K. R. Fuller, Rings and Categories of Modules, Graduate Texts in Mathematics, Vol. 13, Springer-Verlag, New York-Heidelberg, 1974.

[5] D. F. Anderson and P. S. Livingston, The zero-divisor graph of a commutative ring, J. Algebra, 217(2) (1999), 434-447.

[6] H. Ansari-Toroghy and F. Farshadifar, Product and dual product of submodules, Far East J. Math. Sci., 25(3) (2007), 447-455.

[7] H. Ansari-Toroghy and S. Habibi, The Zariski topology-graph of modules over commutative rings, Comm. Algebra, 42(8) (2014), 3283-3296.

[8] H. Ansari-Toroghy and S. Habibi, The annihilating-submodule graph of modules over commutative rings II, Arab. J. Math., 5(4) (2016), 187-194.

[9] H. Ansari-Toroghy and S. Habibi, The annihilating-submodule graph of modules over commutative rings, Math. Rep. (Bucur.), 20(70)(3) (2018), 245-262.

[10] H. Ansari-Toroghy and S. Habibi, The Zariski topology-graph of modules over commutative rings II, Arab. J. Math., 10(1) (2021), 41-50.

[11] M. F. Atiyah and I. G. Macdonald, Introduction to Commutative Algebra, Addison-Wesley Publishing Co., Reading, Mass.-London-Don Mills, Ont., 1969.

[12] R. Balakrishnan and K. Ranganathan, A Textbook of Graph Theory, Universitext, Springer-Verlag, New York, 2000.

[13] I. Beck, Coloring of commutative rings, J. Algebra, 116(1) (1988), 208-226.

[14] M. Behboodi and Z. Rakeei, The annihilating-ideal graph of commutative rings I, J. Algebra Appl., 10(4) (2011), 727-739.

[15] R. Diestel, Graph Theory, Third edition, Graduate Texts in Mathematics, 173, Springer-Verlag, Berlin, 2005. 
[16] T. W. Haynes, S. T. Hedetniemi and P. J. Slater, Domination in Graphs Advanced Topics: Volume 2: Advanced Topics (1st ed.), Routledge, 1998.

[17] T. Y. Lam, A First Course in Noncommutative Rings, Graduate Texts in Mathematics, 131, Springer-Verlag, New York, 1991.

[18] C.-P. Lu, Prime submodules of modules, Comment. Math. Univ. St. Paul., 33(1) (1984), 61-69.

[19] D. A. Mojdeh and A. M. Rahimi, Dominating sets of some graphs associated to commutative rings, Comm. Algebra, 40(9) (2012), 3389-3396.

[20] R. Nikandish, H. R. Maimani and S. Kiani, Domination number in the annihilating-ideal graphs of commutative rings, Publ. Inst. Math. (Beograd) (N.S.), 97(111) (2015), 225-231.

[21] R. Y. Sharp, Steps in Commutative Algebra, London Mathematical Society Student Texts, 19, Cambridge University Press, Cambridge, 1990.

[22] T. Tamizh Chelvam and K. Selvakumar, Central sets in the annihilating-ideal graph of commutative rings, J. Combin. Math. Combin. Comput., 88 (2014) $277-288$.

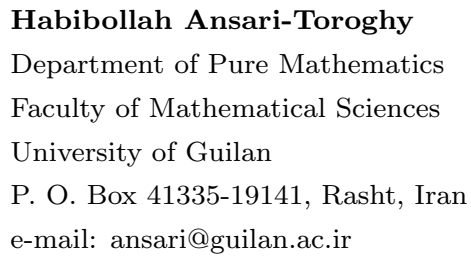

